\title{
Symmetry Analysis of the Fokker Planck Equation
}

\author{
Faya Doumbo Kamano ${ }^{1}$, Bakary Manga ${ }^{2}$, Joël Tossa ${ }^{3}$ \\ ${ }^{1}$ Department of Research, Distance Learning High Institut, Conakry, Guinea \\ ${ }^{2}$ Department of Mathematics and Computer Sciences, University of Cheikh Anta Diop, Dakar, Senegal \\ ${ }^{3}$ Institut of Mathematics and Physical Sciences, University of Abomey-Calavi, Porto-Novo, Benin
}

\section{Email address:}

fd.kamano@isfad-gn.org (F. D. Kamano), bakary.manga@ucad.edu.sn (B. Manga), joel.tossa@imsp-uac.org (J. Tossa)

\section{To cite this article:}

Faya Doumbo Kamano, Bakary Manga, Joël Tossa. Symmetry Analysis of the Fokker Planck Equation. American Journal of Mathematical and Computer Modelling. Vol. 5, No. 2, 2020, pp. 51-60. doi: 10.11648/j.ajmcm.20200502.14

Received: January 30, 2020; Accepted: February 20, 2020; Published: May 28, 2020

\begin{abstract}
In this work, the infinitesimal criterion of invariance for determining symmetries of partial differential equations is applied to the Fokker Planck equation. The maximum rang condition being satisfied, we determine the Lie point symmetries of this equation. Due to the nature of infinitesimal generators of these symmetries and the stability of Lie brackets, we obtain an infinite number of solutions from which we find examples of solutions for the Fokker Planck equation: other solutions are generated given a particular solution of the equation. Then, the Fokker Planck equation admits a conserved form, hence there is an auxiliary system associated to this equation. We show that this system admits six and an infinite number of infinitesimal generators of point symmetries giving rise to two potential symmetries of the Fokker Planck equation. We then use those potential symmetries to determine solutions of the associated system and therefore provide other solutions of the Fokker Planck equation. Note that these are essentially obtained on the basis of the invariant surface conditions. With respect to these conditions and from the potential symmetries that we have found, we finally show that in particular, some solutions of the considered Fokker Planck equation reduced to the trivial solution (solutions that are zero).
\end{abstract}

Keywords: Fokker-Planck Equation, Symmetry Analysis, Lie Point Aymmetry, Potential Symmetry

\section{Introduction}

The Fokker-Planck equation (FPE, for short) is a linear PDE that describes the transition probability density of a Markov process. It is also known as the Kolmogorov diffusion equation and is used to model many situations such as evolution of the distribution function of a particle, finance, turbulence, population dynamics, protein kinetics (see $[3,4,6$, $10,12,21])$.

The FPE interests many researchers as shown by the number of publications on the subject; see e.g. $[2-5,7,8,15$, 16, 19, 20, 21] and references therein.

We state the Fokker-Planck equation (FPE) in the following form:

$u_{t}(x, t)=-a_{2} u(x, t)-\left(a_{2} x+a_{1}\right) u_{x}(x, t)+\frac{1}{2} u_{x x}(x, t)$,

where $a_{1}$ and $a_{2}$ are real numbers; $u(x, t)$ is a function that depends on the variables $x$ and $t$, to be determined; and $u_{\alpha}$ denotes differentiation of $u$ with respect to the variable $\alpha$.
Like most PDEs, it gives explicit solutions only in very specific cases related both to the form of the equation and the shape of the area where it is studied. Many techniques are used to solve particular cases of the FPE: quantum mechanics technique ([2]), Fourier transform method ([19]), differential transform method ([7]), numerical method (e.g. [3, 4, 8, 22]).

Powerful means used in the study of DEs and PDEs are the Lie symmetries. Since their introduction by Sophus Lie ([11]), Lie symmetries are experiencing a rapid development as a wonderful tool for the classification of invariant solutions of DEs and PDEs. Point symmetries are local symmetries as their infinitesimals depend on independent variables $x$ 's, dependent variables $u(x)$ 's, and derivatives of dependent variables; and are determined if $u(x)$ is sufficiently smooth in some neighbourhood of $x$. Potential symmetries when with them are nonlocal symmetries whose infinitesimals, at any point $x$, depend on the global behavior of $u(x)$. Potential symmetries are very useful as they lead to the construction of solutions of a given system of PDEs which cannot be obtained as invariant solutions of its local symmetries. See Section 3 for wider discussion on potential 
symmetries. See also Chap 7 of [1] for more about potential symmetries.

The FPE is considered in his standard expression $[15,18]$ in the form $u_{t}=u+x u_{x}+u_{x x}$ which is different from (1). The authors of the papers quoted above have determined the Lie point symmetries of the FPE, as well as the potential symmetries. They also have provided families of solutions of the FPE.

In this paper we consider the FPE (1) with the condition $a_{2} \neq 0$. We adopt the same approach as in [15] and determine the Lie point symmetries of the FPE in Section 2. Some of its solutions are also determined. In Section 3, we show that the FPE can be written in a conserved form. A conserved form leads to auxiliary dependent variables (which are potentials) and then to an auxiliary system of PDEs whose local symmetries are the potential symmetries of the FPE. We determine such symmetries in Section 3 and use them to construct other solutions of the Fokker-Planck equation.

\section{Point Symmetries of the Fokker-Planck Equation}

\subsection{Some basics about Lie point symmetries}

Consider a general system of $n^{\text {th }}$ order DEs admitting $p$ independent variables $x=\left(x_{1}, \ldots, x_{p}\right)$ in $X \simeq \mathbb{R}^{p}$ and $q$ dependent variables $u=\left(u_{1}, \ldots, u_{q}\right)$ in $U \simeq \mathbb{R}^{q}$,

$$
\Delta_{v}\left(x, u^{(n)}\right)=0, v=1, \ldots, m,
$$

with $u^{(n)}$ denoting the derivatives of the $u$ 's with respect to the $x$ 's up to order $n$. The system (2) is thus defined by the vanishing of a collection of differentiable functions $\Delta_{v}\left(J^{n} \rightarrow \mathbb{R}\right)$ defined on the $n^{\text {th }}$ jet space $J^{n}=J^{n} E=X \times$ $U^{(n)}$, where $E$ is the total space $E=X \times U$ (see [14]). The points in the vertical space $U^{(n)}$ are denoted by $u^{(n)}$ and consist of all the dependent variables and their derivatives up to order $n$. The system (2) can therefore be viewed as defining (or defined by) a variety $\mathrm{S}_{\Delta}=\left\{\left(x, u^{(n)}\right) /\right.$ $\left.\Delta_{v}\left(x, u^{(n)}\right)=0, v=1, \ldots, m\right\}$ contained in the $n^{\text {th }}$ order jet space, and consisting of all points $\left(x, u^{(n)}\right) \in J^{n}$ satisfying the system. The defining functions $\Delta_{v}$ are assumed to be regular in a neighbourhood of $S_{\Delta}$; in particular, this is the case if the Jacobian matrix of the functions $\Delta_{v}$ with respect to the jet variables $\left(x, u^{(n)}\right)$ has maximal rank $m$ everywhere on $S_{\Delta}$. In the case of point transformations, the infinitesimal generators form a Lie algebra $\mathcal{G}$ consisting of vector fields $V=\sum_{i=1}^{p} \xi^{i}(x, u) \frac{\partial}{\partial x^{i}}+\sum_{\alpha=1}^{q} \eta^{\alpha}(x, u) \frac{\partial}{\partial u^{\alpha}} \quad$ on the space of independent and dependent variables.

Let $V^{(n)}$ denote the $n^{\text {th }}$ prolongation of $V$ to the jet space $J^{n}$ ([13, p. 117]):

$$
V^{(n)}=\sum_{i=1}^{p} \xi^{i}(x, u) \frac{\partial}{\partial x^{i}}+\sum_{\alpha=1}^{q} \sum_{H=j=0}^{n} \eta_{J}^{\alpha}\left(x, u^{(j)}\right) \frac{\partial}{\partial u_{J}^{\alpha}},
$$

For any unordered multi-index $J=\left(j_{1}, \ldots, j_{k}\right), 1 \leq j_{k} \leq p$ of order $k=\# \mathrm{~J}=\mathrm{j}_{1}+\ldots+j_{k} \leq n$; where, for any $\alpha=1, \ldots, q$,

$$
\eta_{J}^{\alpha}=D_{J} Q^{\alpha}+\sum_{i=1}^{p} \xi^{i} u_{J, i}^{\alpha} \text { and } Q^{\alpha}=\eta^{\alpha}(x, u)-\sum_{i=1}^{p} \xi^{i}(x, u) \frac{\partial u^{\alpha}}{\partial x^{i}}
$$

The fundamental infinitesimal symmetry criterion for the system (2) is stated in the following:

Theorem 2.1 ([14]). A connected group of transformations $G$ is a symmetry group of the fully regular system of DEs (2) if and only if the infinitesimal symmetry conditions

$$
V^{(n)}\left(\Delta_{v}\right)=0, v=1, \ldots, m, \text { whenever } \Delta=0,
$$

hold for every infinitesimal generator $V$ of the Lie algebra $g$ of $G$.

Let $u=f(x)=f\left(x_{1}, \ldots, x_{p}\right)$ be a function of $\mathbb{R}^{p}$ with values in $\mathbb{R} .$. It is known that there exists $p_{r}=\left(\begin{array}{l}p+r-1 \\ p\end{array}\right)$ derivatives of $f$ of order $r$. The equation $\Delta\left(x, u^{(n)}=0\right.$ is defined on the space $\mathbb{R}^{p} \times U^{n}$ of dimension $p+q p^{(n)}$, with $p^{(n)}=1+p_{1}+p_{2}+\ldots+p_{n}=\left(\begin{array}{c}p+n \\ n\end{array}\right)$.

A system $\Delta\left(x, u^{(n)}\right)=\left(\Delta_{1}\left(x, u^{(n)}\right), \ldots, \Delta_{m}\left(x, u^{(n)}\right)\right)$ will have as Jacobian matrix, a matrix of rank $m \times\left(p+q p^{(n)}\right)$. See more details in [13, p. 95].
Definition 2.2 ([12]). The system (2) is said to be of maximum rank if the $m \times\left(p+q p^{(n)}\right)$ Jacobian matrix $J_{\Delta}\left(x, u^{(n)}\right):=\left(\frac{\partial \Delta_{v}}{\partial x^{i}}, \frac{\partial \Delta_{v}}{\partial u_{J}^{\alpha}}\right)$ of $\Delta$ with respect to all the variables $\left(x, u^{(n)}\right)$ is of rank $m$ whenever $\Delta\left(x, u^{(n)}\right)=0$.

\subsection{Lie Point Symmetries of the FPE}

To investigate the Lie point symmetries of the FPE, we have to check the maximal rank condition for the map $\Delta:\left(x, t ; u^{(2)}\right) \mapsto u_{t}(x, t)+a_{2} u(x, t)+\left(a_{2} x+a_{1}\right) u_{x}(x, t)-\frac{1}{2} u_{x x}(x ; t)$

whose kernel equation is (1) on a subset $M^{(2)}$ of the $2^{\text {nd }}$ jetspace $X \times U^{(2)}$ of the manifold $X \times U$. The independent variables $(x, t)$ and the dependent variable leave on the spaces $U \simeq \mathbb{R}^{2}$ and $U \simeq \mathbb{R}$, respectively. The expression $u^{(2)}=\left(u, u_{x}, u_{t}, u_{x x}, u_{x t}, u_{t t}\right)$ represents the various partial derivatives up to the second order of $u$, and leaves on the second prolongation $U^{(2)}$ of the set $U$. The set $M^{(2)}$ is the corresponding $2^{\text {nd }}$ prolongation of the subspace $M \subset X \times U$. 
The Jacobian matrix of $\Delta$, $J_{\Delta}\left(x, t ; u^{(2)}\right)=\left(a_{2} u_{x}, 0, a_{2}, a_{1}+a_{2} x, 1,-\frac{1}{2}, 0,0\right)$ does not vanish anywhere on $M^{(2)}$. Then, $\Delta$ is of maximal rank. Let

$$
V^{(2)}=V+\eta^{x}\left(x, t, u^{(2)}\right) \frac{\partial}{\partial u_{x}}+\eta^{t}\left(x, t, u^{(2)}\right) \frac{\partial}{\partial u_{t}}+\eta^{x x}\left(x, t, u^{(2)}\right) \frac{\partial}{\partial u_{x x}}+\eta^{x t}\left(x, t, u^{(2)}\right) \frac{\partial}{\partial u_{x t}}+\eta^{t t}\left(x, t, u^{(2)}\right) \frac{\partial}{\partial u_{t t}},
$$

Where $\eta^{x}, \eta^{t}, \eta^{x x}, \eta^{x t}$ and $\eta^{t t}$ are given by the formulae (see [14]):

$$
\begin{gathered}
\eta^{x}=D_{x}\left(\eta-\xi u_{x}-\tau u_{t}\right)+\xi u_{x x}+\tau u_{x t}, \\
\eta^{t}=D_{t}\left(\eta-\xi u_{x}-\tau u_{t}\right)+\xi u_{t x}+\tau u_{t t}, \\
\eta^{x x}=D_{x x}\left(\eta-\xi u_{x}-\tau u_{t}\right)+\xi u_{x x x}+\tau u_{x x t}, \\
\eta^{x t}=D_{x t}\left(\eta-\xi u_{x}-\tau u_{t}\right)+\xi u_{x x t}+\tau u_{t t x}, \\
\eta^{t t}=D_{t t}\left(\eta-\xi u_{x}-\tau u_{t}\right)+\xi u_{t t x}+\tau u_{t t t} .
\end{gathered}
$$

Proposition 2.3.: Point symmetries of the FPE are generated by the operators

$$
\begin{gathered}
V_{1}=e^{a_{2} t} \frac{\partial}{\partial x}, V_{2}=u \frac{\partial}{\partial u}, V_{4}=\frac{\partial}{\partial t}, \\
V_{3}=\frac{1}{2 a_{2}} e^{-a_{2} t} \frac{\partial}{\partial x}+\left(a_{2} x+a_{1}\right) \frac{u}{a_{2}} e^{-a_{2} t} \frac{\partial}{\partial u}, \\
V_{5}=e^{-2 a_{2} t} \frac{\partial}{\partial t}-\left(a_{2} x+a_{1}\right) e^{-2 a_{2} t} \frac{\partial}{\partial x}-2\left(a_{2} x+a_{1}\right)^{2} e^{-2 a_{2} t} u \frac{\partial}{\partial u}, \\
V_{6}=e^{2 a_{2} t} \frac{\partial}{\partial t}+\left(a_{2} x+a_{1}\right) e^{2 a_{2} t} \frac{\partial}{\partial x}-a_{2} u e^{2 a_{2} t} \frac{\partial}{\partial u},
\end{gathered}
$$

and an infinite number of generators $V_{\alpha}=\alpha(x, t) \frac{\partial}{\partial u}$; where $\alpha$ is any solution of the FPE.

Proof. We make the assumption $V^{(2)} \Delta\left(x, t ; u^{(2)}\right)=0$ whenever, and check the corresponding conditions on $\xi, \tau$ and $\eta$. Those conditions lead to

$$
\left(\eta^{t}=-a_{2} \eta-a_{1} \eta^{x}-a_{2} \xi u_{x}-a_{2} x \eta^{x}+\frac{1}{2} \eta^{x x}\right)_{\Delta=0}
$$

Now replace $\eta^{x}, \eta^{t}$ and $\eta^{x x}$ in (16) by their expressions given in (7), (8) and (9) respectively, and eliminate $u_{t}$ by substituting it by the right hand side of (1) any time when it occurs. Then the derivatives of $u$ with right to $t$ disappear. So, the resolution of the corresponding system of PDEs is equivalent to solving the following system:

$$
\begin{gathered}
\eta_{u u}=0 \\
2 \xi_{x}-\tau_{t}=0, \tau_{x}=0, \tau_{u}=0, \xi_{u}=0,
\end{gathered}
$$

$V=\xi(x, t, u) \frac{\partial}{\partial x}+\tau(x, t, u) \frac{\partial}{\partial t}+\eta(x, t, u) \frac{\partial}{\partial u}$ be a vector field on $X \times M$, where $\xi, \tau$ and $\eta$ are smooth functions. The second prolongation of $V$ reads

$$
\begin{gathered}
2\left(a_{2} x+a_{1}\right) \xi_{x}-2 \xi_{t}+2 a_{2} \xi-2 \eta_{x u}=0, \\
\eta_{x x}-2\left(a_{2} x+a_{1}\right) \eta_{x}-2 \eta_{t}+2 a_{2} u \eta_{u}-2 a_{2} \eta-2 a_{2} u \tau_{t}=0,
\end{gathered}
$$

Equation (17) implies that $\eta$ is linear in $u$. So, it writes

$$
\eta(x, t, u)=A(x, t) u+B(x, t),
$$

$A$ and $B$ being smooth functions depending only on $x$ and $t$. From (18), we get

$$
\xi=\frac{1}{2} \tau_{t} x+k(t)
$$

where $k$ is a smooth function of $t$. Substituting $\xi$ and $\eta$ by their expressions in (19) and differentiating the resulting expression with respect to $x$, we get $2 A_{x x}-2 a_{2} \tau_{t}+\tau_{t t}=0$. Thus,

$$
\begin{gathered}
A(x, t)=\left(\frac{1}{2} a_{2} \tau_{t}-\frac{1}{4} \tau_{t t}\right) x^{2}+A_{1}(t) x+A_{2}(t), \\
k^{\prime}(t)-a_{2} k(t)+A_{1}(t)-\frac{1}{2} a_{1} \tau_{t}=0,
\end{gathered}
$$

where $A_{1}$ and $A_{2}$ are smooth functions of the variable $t$. Using Equation (20), we find that

$$
\begin{gathered}
A_{x x}-2 a_{2} x A_{x}-2 a_{1} A_{x}-2 A_{t}-2 a_{2} \tau_{t}=0, \\
-\frac{1}{2} B_{x x}+a_{2} x B_{x}+a_{1} B_{x}+a_{2} B+B_{t}=0 .
\end{gathered}
$$

Note that (22) is nothing but the FPE (1). Now (19), (20) and (21) entail

$$
\begin{gathered}
\tau(t)=C_{1} e^{2 a_{2} t}+C_{2} e^{-2 a_{2} t}+C_{3} \\
A_{1}(t)=\left[-4 a_{1} a_{2} C_{2} e^{-a_{2} t}+C_{4}\right] e^{-a_{2} t}, \\
A_{2}(t)=-2 a_{1}^{2} C_{2} e^{-2 a_{2} t}+\frac{1}{a_{2}} C_{4} a_{1} e^{-a_{2} t}-C_{1} a_{2} e^{2 a_{2} t}+C_{5}, \\
k(t)=a_{1} C_{1} e^{a_{2} t}-a_{1} C_{2} e^{-2 a_{2} t}+\frac{C_{4}}{2 a_{2}} e^{-a_{2} t}+C_{6} e^{a_{2} t},
\end{gathered}
$$

where $C_{1}, C_{2}, \ldots, C_{6}$ are real numbers. Hence, the solution of the system (17)-(20) is 


$$
\begin{gathered}
\xi(x, t, u)=\left[C_{1} a_{2} e^{2 a_{2} t}-C_{2} a_{2} e^{-2 a_{2} t}\right] x+a_{1} C_{1} e^{2 a_{2} t}-a_{1} C_{2} e^{-2 a_{2} t}+\frac{C_{4}}{2 a_{2}} e^{-a_{2} t}+C_{6} e^{a_{2} t} \\
\tau(x, t, u)=C_{1} e^{2 a_{2} t}+C_{2} e^{-2 a_{2} t}+C_{3} \\
\eta(x, t, u)=\left(-2 C_{2}\left(a_{2} x+a_{1}\right)^{2} e^{-2 a_{2} t}+\frac{C_{4}}{a_{2}}\left(a_{2} x+a_{1}\right) e^{-a_{2} t}\right) u-C_{1} a_{2} e^{2 a_{2} t} u+C_{5} u+\alpha(x, t)
\end{gathered}
$$

where $\alpha(x, t)=B(x, t)$ is any solution of the FPE. The rest of the proof is straightforward.

\subsection{Examples of solutions of the FPE}

In the sequel, we provide a family of solutions of the Fokker-Planck equation (1).

Theorem 2.4. Let $\alpha(x, t)$ be any solution of the FPE. Then the functions

$$
\begin{gathered}
f_{1}(x, t)=e^{-2 a_{2} t}\left[\alpha_{t}-\left(a_{2} x+a_{1}\right) \alpha_{x}+2\left(a_{2} x+a_{1}\right)^{2} \alpha\right], \\
f_{2}(x, t)=\frac{e^{-a_{2} t}}{a_{2}}\left(\frac{1}{2} \alpha_{x}-\left(a_{2} x+a_{1}\right) \alpha\right), \\
f_{3}(x, t)=e^{2 a_{2} t}\left[\alpha_{t}+\left(a_{2} x+a_{1}\right) \alpha_{x}+a_{2} \alpha\right], \\
f_{4}(x, t)=\alpha_{x} e^{a_{2} t}, f_{5}(x, t)=\alpha_{t}
\end{gathered}
$$

\begin{tabular}{|c|c|c|}
\hline$\left[V_{1}, V_{2}\right]=0$ & {$\left[V_{2}, V_{4}\right]=0$} & {$\left[V_{3}, V_{\alpha}\right]=V_{\frac{e^{-a r y}}{a_{2}}\left[\frac{1}{2} \alpha_{2},-\left(a_{2} x+a_{1}\right) \alpha\right]}$} \\
\hline$\left[V_{1}, V_{3}\right]=V_{2}$ & {$\left[V_{2}, V_{5}\right]=0$} & {$\left[V_{4}, V_{5}\right]=-2 a_{2} V_{5}$} \\
\hline$\left[V_{1}, V_{4}\right]=-a_{2} V_{1}$ & {$\left[V_{2}, V_{6}\right]=0$} & {$\left[V_{4}, V_{6}\right]=2 a_{2} V_{6}$} \\
\hline$\left[V_{1}, V_{5}\right]=-4 a_{2}^{2} V_{3}$ & {$\left[V_{2}, V_{\alpha}\right]=-V_{\alpha}$} & {$\left[V_{4}, V_{\alpha}\right]=V_{\alpha_{i}}$} \\
\hline$\left[V_{1}, V_{6}\right]=0$ & {$\left[V_{3}, V_{4}\right]=a_{2} V_{3}$} & {$\left[V_{5}, V_{6}\right]=4 a_{2} V_{4}-2 a_{2}^{2} V_{2}$} \\
\hline$\left[V_{1}, V_{\alpha}\right]=V_{\alpha_{e} e^{e t}}$ & {$\left[V_{3}, V_{5}\right]=0$} & {$\left[V_{5}, V_{\alpha}\right]=V_{e^{-2 x_{2}}[}\left[\alpha_{1}-\left(a_{2} x+a_{1}\right) \alpha_{x}+2\left(a_{2} x+a_{1}\right)^{2} \alpha\right]$} \\
\hline$\left[V_{2}, V_{3}\right]=0$ & {$\left[V_{3}, V_{6}\right]=V_{1}$} & {$\left[V_{6}, V_{\alpha}\right]=V_{e^{2 a_{2} a_{2}}\left[\alpha_{1}+\left(a_{2} x+a_{1}\right) \alpha_{x}+a_{2} \alpha\right]}$} \\
\hline
\end{tabular}

are also solutions of the FPE.

Proof. Since $\left\{V_{\alpha}, V_{i}, i=1, \ldots, 6\right\}$ generates a Lie algebra, the stability of the brackets in the table below completes the proof.
Table 1. Commutations table of the Lie algebra of symmetries of the FPE.

As mentioned in [15], using the Lie brackets in Table 1, one can construct a family of solutions from a trivial solution. Consider e.g. $u(x, t)=e^{-a_{2} t}$ then the functions

$$
\begin{gathered}
g_{1}(x, t)=\left[-a_{2}+2\left(a_{2} x+a_{1}\right)^{2}\right] e^{-3 a_{2} t}, \\
g_{2}(x, t)=-\left(x+\frac{a_{1}}{a_{2}}\right) e^{-2 a_{2} t}, \\
g_{3}(x, t)=-a_{2} e^{-a_{2} t}
\end{gathered}
$$

are also solutions of (1). From these solutions we can again construct other solutions. For instance, applying the symmetry generators (35) to $g_{1}$ yields to the solution

$$
g_{4}(x, t)=\left[3 a_{2}^{2}-12 a_{2}\left(a_{2} x+a_{1}\right)^{2}+4\left(a_{2} x+a_{1}\right)^{4}\right] \exp \left(-5 a_{2} t\right)
$$

\section{Potential Symmetries of the FPE}

\subsection{Preliminaries on Potential Symmetries}

A partial differential equation of order $n$ in the unknown function $u(x, t)$

$$
\Delta\left(x, t, u^{(n)}\right)=0
$$

is written in a conserved form if it has the following form:

$$
\begin{aligned}
& D_{t} T\left(x, t, u^{(n-1)}\right)+D_{x} X\left(x, t, u^{(n-1)}\right)=0 \\
& W=\xi(x, t, u, v) \frac{\partial}{\partial x}+\tau(x, t, u, v) \frac{\partial}{\partial t}+\eta(x, t, u, v) \frac{\partial}{\partial u}+\varphi(x, t, u, v) \frac{\partial}{\partial v} .
\end{aligned}
$$

One says that $S\left(x, t, u^{(n-1)} v_{x}, v_{t}\right)$ defines a potential symmetry admitted by (42) if and only if one, at least, of the infinitesimals $\xi, \tau$ and $\eta$ depends explicitly on the potential $v$; that is if and only if the condition
Since the PDE (43) is in a conserved form, a potential $v$ considered as a new variable is in-traduced. A system of PDEs denoted by $S\left(x, t, u^{(n-1)}, v_{x}, v_{t}\right)$ is then obtained. If $(u(x, t), v(x, t))$ is a solution of the system $S\left(x, t, u^{(n-1)}, v_{x}, v_{t}\right)$, then $u(x, t)$ solves the PDE given by (42).

Definition 3.1. Assume that the auxiliary system $S\left(x, t, u^{(n-1)}, v_{x}, v_{t}\right)$ admits a generator $W$ of point symmetries given by 
$Y=\xi(x, t, u, v) \frac{\partial}{\partial x}+\tau(x, t, u, v) \frac{\partial}{\partial t}+\eta(x, t, u ; v) \frac{\partial}{\partial u} \quad$ will $\quad$ be called a potential symmetry of Equation (42).

Potential symmetries can also be used in the study of a boundary value problem posed for a given system of PDEs and for the study of ODEs. For a scalar ODE, a potential symmetry reduces the order (see [1]).

$\mathrm{We}$ are now going to explain how, from potential symmetries, one obtains solutions of the PDE (42) which admits a conserved form (43). See [18] for wider discussion. Given point symmetry $\xi \frac{\partial}{\partial x}+\tau \frac{\partial}{\partial t}+\eta \frac{\partial}{\partial u}+\varphi \frac{\partial}{\partial v}$ of (43), the invariant surface conditions are

$$
\begin{gathered}
\xi(x, t, u, v) u_{x}+\tau(x, t, u, v) u_{t}-\eta(x, t, u, v)=0 \\
\xi(x, t, u, v) v_{x}+\tau(x, t, u, v) v_{t}-\varphi(x, t, u, v)=0
\end{gathered}
$$

The associated characteristic system yields to the following independent integrals

$$
S_{1}(x, t, u, v)=C_{1}, S_{2}(x, t, u, v)=C_{2}, S_{3}(x, t, u, v)=C_{3},
$$

with $\frac{\partial\left(S_{1}, S_{2}, S_{3}\right)}{\partial(u, v)}$ of rank 2. If we set $z=c_{1}, c_{2}=h_{1}(z)$ and $c_{3}=h_{2}(z)$, we obtain from (47):

$$
u=U\left(x, t, z, h_{1}(z), h_{2}(z)\right)
$$

$$
\begin{aligned}
& v=V\left(x, t, z, h_{1}(z), h_{2}(z)\right), \\
& G\left(x, t, z, h_{1}(z), h_{2}(z)\right)=0 .
\end{aligned}
$$

The invariant solutions of (43) are given by (48) and (49), where $h_{i}(z)$ are the solutions of the ordinary system obtained by substitution in (43). Since (42) is a differential consequence of (43), the solution of (43) give those solutions of (42), which verify the differential relation obtained by eliminating $v$ between (45) and $\xi T+\tau X-\varphi=0$.

\subsection{Potential symmetries of the FPE}

The conserved form of the FPE can be written as

$$
D_{t} u+D_{x}\left(-\left(a_{2} x+a_{1}\right) u+\frac{1}{2} u_{x}\right)=0 .
$$

Then, the corresponding system writes as follows:

$$
\left\{\begin{array}{l}
v_{t}=-\left(a_{2} x+a_{1}\right) u+\frac{1}{2} u_{x} \\
v_{x}=u
\end{array}\right.
$$

where the potential variable $v$ has been introduced as a new dependent variable.

Proposition 3.2. The system (52), with $a_{1} \in \mathbb{R}$ and, $a_{2} \neq 0$ admits a non trivial symmetry group with the following infinitesimal generators:

$$
\begin{gathered}
W_{1}=e^{a_{2} t} \frac{\partial}{\partial x}, W_{2}=u \frac{\partial}{\partial u}+v \frac{\partial}{\partial v}, W_{3}=\frac{\partial}{\partial t}, \\
W_{4}=\frac{1}{2 a_{2}} e^{-a_{2} t} \frac{\partial}{\partial x}+\left[\left(x+\frac{a_{1}}{a_{2}}\right) u+V\right] e^{-a_{2} t} \frac{\partial}{\partial u}+\left(\frac{a_{1}}{a_{2}}+x V\right) e^{-a_{2} t} \frac{\partial}{\partial v}, \\
W_{5}=-\left(a_{2} x+a_{1}\right) e^{-2 a_{2} t} \frac{\partial}{\partial x}+e^{-2 a_{2} t} \frac{\partial}{\partial t}-2\left[\left(\left(a_{2} x+a_{1}\right)^{2}-a_{2}\right) u+2 a_{2}\left(a_{2} x+a_{1}\right) V\right] e^{-2 a_{2} t} \frac{\partial}{\partial u} \\
-2\left[\left(a_{2} x+a_{1}\right)^{2}-a_{2}\right] v e^{-2 a_{2} t} \frac{\partial}{\partial v} \\
W_{6}=e^{2 a_{2} t} \frac{\partial}{\partial t}+\left(a_{2} x+a_{1}\right) e^{2 a_{2} t} \frac{\partial}{\partial x}-a_{2} u e^{2 a_{2} t} \frac{\partial}{\partial u},
\end{gathered}
$$

and an infinite number of generators of the form $W_{\beta}=\beta_{x}(x, t) \frac{\partial}{\partial u}+\beta(x, t) \frac{\partial}{\partial v}$, where $\beta(x, t)$ satisfies the equation $\beta_{t}=-\left(a_{2} x+a_{1}\right) \beta_{x}+\frac{1}{2} \beta_{x x}$

Proof. Let $\Delta_{1}=v_{t}+\left(a_{2} x+a_{1}\right) u-\frac{1}{2} u_{x}$ and $\Delta_{2}=v_{x}-u$ be the associated system to the system (52) and let $W=\xi(x, t, u, v) \frac{\partial}{\partial x}+\tau(x, t, u, v) \frac{\partial}{\partial t}+\eta(x, t, u, v) \frac{\partial}{\partial u}+\varphi(x, t, u, v) \frac{\partial}{\partial v}$ be a symmetry vector field of this system. The criterion (5) writes $\left.W^{(2)}\left(\Delta_{i}\right)\right|_{\Delta i=0, i=1,2}=0$, where 


$$
\begin{aligned}
& W^{(2)}=W+\eta^{x} \frac{\partial}{\partial u_{x}}+\eta^{t} \frac{\partial}{\partial u_{t}}+\varphi^{x} \frac{\partial}{\partial v_{x}}+\varphi^{t} \frac{\partial}{\partial v_{t}}+\eta^{x x} \frac{\partial}{\partial u_{x x}}+\eta^{x t} \frac{\partial}{\partial u_{x t}} \\
& +\eta^{t t} \frac{\partial}{\partial u_{t t}}+\varphi^{x x} \frac{\partial}{\partial v_{x x}}+\varphi^{x t} \frac{\partial}{\partial v_{x t}}+\varphi^{t t} \frac{\partial}{\partial v_{t t}} .
\end{aligned}
$$

The coefficient functions $\left(\eta^{x}, \eta^{t}, \varphi^{x}, \varphi^{t}, \eta^{x x}, \eta^{x t}, \eta^{t t}, \varphi^{x x}, \varphi^{x t}, \varphi^{t t}\right)$ in $W^{(2)}$ are given as follows:

$$
\begin{gathered}
\eta^{x}=D_{x} \eta-u_{x} D_{x} \xi-u_{t} D_{x} \tau, \eta^{t}=D_{t} \eta-u_{x} D_{t} \xi-u_{t} D_{t} \tau, \\
\varphi^{x}=D_{x} \varphi-v_{x} D_{x} \xi-v_{t} D_{x} \tau, \varphi^{t}=D_{t} \varphi-v_{x} D_{t} \xi-v_{t} D_{t} \tau, \\
\eta^{x x}=D_{x}^{2} \eta-u_{x} D_{x}^{2} \xi-u_{t} D_{x}^{2} \tau-2 u_{x x} D_{x} \xi-2 u_{x t} D_{x} \tau, \\
\varphi^{x x}=D_{x}^{2} \varphi-v_{x} D_{x}^{2} \xi-v_{t} D_{x}^{2} \tau-2 v_{x x} D_{x} \xi-2 v_{x t} D_{x} \tau, \\
\eta^{x t}=D_{x t}^{2} \eta-u_{x} D_{x t}^{2} \xi-u_{t} D_{x t}^{2} \tau-u_{x x} D_{t} \xi-u_{t x} D_{t} \tau-u_{x t} D_{x} \xi-u_{t t} D_{x} \tau, \\
\varphi^{x t}=D_{x t}^{2} \varphi-v_{x} D_{x t}^{2} \xi-v_{t} D_{x t}^{2} \tau-v_{x x} D_{t} \xi-v_{t x} D_{t} \tau-v_{x t} D_{x} \xi-v_{t t} D_{x} \tau, \\
\eta^{t t}=D_{t}^{2} \eta-u_{x} D_{t}^{2} \xi-u_{t} D_{t}^{2} \tau-2 u_{x t} D_{t} \xi-2 u_{t t} D_{t} \tau, \\
\varphi^{t t}=D_{t}^{2} \varphi-v_{x} D_{t}^{2} \xi-v_{t} D_{t}^{2} \tau-2 v_{x t} D_{t} \xi-2 v_{t t} D_{t} \tau
\end{gathered}
$$

Hence, the criterion $\left.W^{(2)}\left(\Delta_{i}\right)\right|_{\Delta_{i}=0, i=1,2}=0$ gives the following equalities:

$$
\begin{gathered}
\left(\varphi^{t}+a_{2} \xi u+\left(a_{2} x+a_{1}\right) \eta-\frac{1}{2} \eta^{x}=0\right)_{\mid \Delta_{i}=0, i=1,2} \\
\left(\varphi^{x}-\eta=0\right)_{\mid \Delta_{i}=0, i=1,2}
\end{gathered}
$$

Replacing $\varphi^{x}, \varphi^{t}$ and $\eta^{x}$ by their expressions in (66)-(67) and equalizing the coefficients of the remaining unconstrained partial derivatives of $u$ and $v$ to zero, one obtains:

$$
\begin{gathered}
\xi_{u}=0, \xi_{v}=0,2 \xi_{x}=\tau_{t}, \\
\tau_{x}=0, \tau_{u}=0, \tau_{v}=0, \\
\varphi_{u}=0, \varphi_{v v}=0, \\
\tau_{t t}=4 a_{2}^{2} \tau_{t}, 2 \xi_{t t}=3 a_{2}\left[\left(a_{2} x+a_{1}\right) \tau_{t}+\frac{2}{3} a_{2} \xi\right], \\
2 \eta=2 \varphi_{x}-\tau_{t} u+2 u \varphi_{v}, \\
2 \varphi_{v x}=\left(a_{2} x+a_{1}\right) \tau_{t}-2 \xi_{t}+2 a_{2} \xi, \\
\varphi_{x x}=2\left(a_{2} x+a_{1}\right) \varphi_{x}+2 \varphi_{t}, \\
4 \varphi_{t v}=-\tau_{t t}+\left(-2 a_{2}^{2} x^{2}+\left(2-4 a_{1} x\right) a_{2}-2 a_{1}^{2}\right) \tau_{t}+\left(a_{2} x+a_{1}\right) \xi_{t}-a_{2}\left(a_{2} x+a_{1}\right) \xi .
\end{gathered}
$$

Equations (69) imply that $\tau$ depends only on $t$. Hence, relations in (68) yield to

$$
\xi=\frac{1}{2} \tau_{t} x+L(t)
$$


where $L$ is a smooth function of $t$. Relations (70) imply that $\varphi$ is independent from $u$ and is linear with right to $v$. That is there exists functions $D$ and $E$ depending only on $x$ and $t$ such that

$$
\varphi=D(x, t) v+E(x, t)
$$

Then, substituting $\xi$ and $\varphi$ by their expressions in (73) and differentiating the resulting expression with respect to $x$, one obtains the equation $2 D_{x x}-2 a_{2} \tau_{t}+\tau_{t t}=0$. Thus

$$
\begin{gathered}
D(x, t)=\left(\frac{1}{2} a_{2} \tau_{t}-\frac{1}{4} \tau_{t t}\right) x^{2}+B_{1}(t) x+B_{2}(t), \\
L^{\prime}(t)-a_{2} L(t)+B_{1}(t)-\frac{1}{2} a_{1} \tau_{t}=0,
\end{gathered}
$$

where $B_{1}$ and $B_{2}$ are smooth functions of $t$ only. Coming back to Equation (74), we find that

$$
\begin{gathered}
D_{x x}-2\left(a_{2} x-a_{1}\right) D_{x}-2 D_{t}=0, \\
E_{x x}-2\left(a_{2} x-a_{1}\right) E_{x}-2 E_{t}=0 .
\end{gathered}
$$

Here again, (81) is equivalent to (52). From Equations (78) and (80), one gets

$$
\begin{gathered}
\tau(t)=C_{1} e^{2 a_{2} t}+C_{2} e^{-2 a_{2} t}+C_{3}, \\
B_{1}(t)=\left(-4 a_{1} a_{2} C_{2} e^{-a_{2} t}+C_{4}\right) e^{-a_{2} t}, \\
B_{2}(t)=-2 a_{1}^{2} C_{2 e^{-2 a_{2} t}}+\frac{1}{a_{2}} C_{4} a_{1} e^{-a_{2} t}+C_{2} a_{2} e^{-2 a_{2} t}+C_{5}
\end{gathered}
$$

where $C_{1}, C_{2}, C_{3}, C_{4}$ and $C_{5}$ are arbitrary constants. Now, Equation (79) yields:

$$
L(t)=a_{1} C_{1} e^{2 a_{2} t}+a_{1} C_{2} e^{-2 a_{2} t}+\frac{C_{4}}{2 a_{2}} e^{-a_{2} t}+C_{6} e^{a_{2} t},
$$

where $C_{6}$ is an arbitrary constant. Hence, expressions (72), (76) and (77) read:

$$
\begin{gathered}
\xi=C_{1}\left(a_{2} x+a_{1}\right) e^{2 a_{2} t}-C_{2}\left(a_{2} x+a_{1}\right) e^{-2 a_{2} t}+C_{4} \frac{e^{-a_{2} t}}{2 a_{2}}+C_{6} e^{a_{2} t} \\
\varphi=-2 C_{2}\left[\left(a_{2} x+a_{1}\right)^{2}-a_{2}\right] v e^{-2 a_{2} t}+C_{4}\left(x+\frac{a_{1}}{a_{2}}\right) v e^{-a_{2} t}+C_{5} v+\beta(x, t), \\
\eta=-C_{1} a_{2} u e^{2 a_{2} t}-2 C_{2}\left[\left(\left(a_{2} x+a_{1}\right)^{2}-a_{2}\right) u+2 a_{2}\left(a_{2} x+a_{1}\right) v\right] e^{-2 a_{2}} \\
+C_{4}\left[v+\left(x+\frac{a_{1}}{a_{2}}\right) u\right] e^{-a_{2} t}+C_{5} u+\beta_{x}(x, t)
\end{gathered}
$$

where $C_{1}, \ldots, C_{6}$ are arbitrary constants and $\beta(x, t)=E(x, t)$ is any solution of $(81)$. It is now a little matter to complete the proof.

It is readily verified that $W_{3}$ and $W_{5}$ in Proposition 3.2 are the only generators of the point symmetries of the system (51) that satisfy condition (44). Hence, we have the

Proposition 3.3. The potential symmetries of the FPE are generated by the vector fields

$$
Y_{1}=\frac{1}{2 a_{2}} e^{-a_{2} t} \frac{\partial}{\partial x}+\left[\left(x+\frac{a_{1}}{a_{2}}\right) u+v\right] e^{-a_{2} t} \frac{\partial}{\partial u}
$$




$$
Y_{2}=-\left(a_{2} x+a_{1}\right) e^{-2 a_{2} t} \frac{\partial}{\partial x}+e^{-2 a_{2} t} \frac{\partial}{\partial t}-2\left[\left(\left(a_{2} x+a_{1}\right)^{2}-a_{2}\right) u+2 a_{2}\left(a_{2} x+a_{1}\right) v\right] e^{-2 a_{2} t} \frac{\partial}{\partial u}
$$

Consider the Symmetries $W_{3}$ which yields to the potential symmetry $Y_{1}$. The associated invariant surface conditions are

$$
\begin{gathered}
u_{x}-2\left(a_{2} x+a_{1}\right) u-2 a_{2} v=0, \\
v_{x}-2\left(a_{2} x+a_{1}\right) v=0 .
\end{gathered}
$$

The system below admits the following solutions:

$$
u(x, t)=\left[2 a_{2} x q_{1}(t)+q_{2}(t)\right] \exp \left(a_{2} x^{2}+2 a_{1} x\right), v(x, t)=q_{1}(t) \exp \left(a_{2} x^{2}+2 a_{1} x\right),
$$

where $q_{1}$ and $q_{2}$ are smooth functions of the variable $t$. If we replace the expression of $u(x, t)$ given by (93) in (1), we get

$$
2 a_{2}\left[a_{2} q_{1}(t)+q_{1}^{\prime}(t)\right] x+\left[q_{2}^{\prime}(t)-q_{2}(t)\right]=0 .
$$

Hence, $q_{1}^{\prime}(t)+a_{2} q_{1}(t)=0$ and $q_{2}^{\prime}(t)-q_{2}(t)=0$ and this yield to $q_{1}(t)=a e^{-a_{2} t}$ and $q_{2}(t)=b e^{t}$. Then we have the following solution of the FPE (1):

$$
u(x, t)=\left[2 a_{2} a x e^{-a_{2} t}+b e^{t}\right] \exp \left(a_{2} x^{2}+2 a_{1} x\right),
$$

where $a$ and $b$ are constants.

Let us now deal with the symmetry generator $W_{5}$ which provides the potential symmetry $Y_{2}$. The invariant surface conditions for this symmetry write

$$
\begin{gathered}
-\left(a_{2} x+a_{1}\right) u_{x}+u_{t}+2\left[\left(a_{2} x+a_{1}\right)^{2}-a_{2}\right] u+4 a_{2}\left(a_{2} x+a_{1}\right) v=0, \\
-\left(a_{2} x+a_{1}\right) v_{x}+v_{t}+2\left[\left(a_{2} x+a_{1}\right)^{2}-a_{2}\right] v=0 .
\end{gathered}
$$

A solution of Equation (76) writes

$$
v(x, t)=\frac{f\left(\left(a_{2} x+a_{1}\right) e^{a_{2} t}\right)}{\left(a_{2} x+a_{1}\right)^{2}} \exp \left(\frac{\left(a_{2} x+a_{1}\right)^{2}}{a_{2}}\right),
$$

where $f$ is a smooth function. Replacing the expression of $v(x, t)$ given by (98) in (96) and solving the latter, we get

$$
u(x, t)=\frac{4 a_{2} x f\left(\left(a_{2} x+a_{1}\right) e^{a_{2} t}\right)+g\left(\left(a_{2} x+a_{1}\right) e^{a_{2} t}\right)}{\left(a_{2} x+a_{1}\right)^{2}} \exp \left(\frac{\left(a_{2} x+a_{1}\right)^{2}}{a_{2}}\right)
$$

where $g$ is another smooth function. Now, setting $z=\left(a_{2} x+a_{1}\right) e^{a_{2} t}$ and putting expression (99) in the Fokker-Planck equation (1) yields to an equation that can be regarded as the vanishing of a polynomial of degree 3 in $e^{a_{2} t}$. Then, the vanishing of the coefficients of this polynomial leads to the following equations:

$$
\begin{gathered}
\left(1-a_{2}\right) z f^{\prime}(z)+a_{2} f(z)=0, \\
-8 a_{2} a_{1} f(z)+2 a_{2} g(z)+4 z a_{1}\left(a_{2}-1\right) f^{\prime}(z)+z\left(1-a_{2}\right) g^{\prime}(z)=0, \\
2 f(z)-2 z f^{\prime}(z)+z^{2} f^{\prime \prime}(z)=0, \\
24 a_{1} f(z)-6 g(z)-16 a_{1} z f^{\prime}(z)+4 z g^{\prime}(z)+4 a_{1} z^{2} f^{\prime \prime}(z)-z^{2} g^{\prime \prime}(z)=0 .
\end{gathered}
$$

i) If $a_{2}=1$, the solution of the system is $f(z)=g(z)=0$ for all $z$ and we get the trivial solution $u(x, t)=0$ for all $x$ and $t$. 
ii) Suppose $a_{2} \neq 1$. Then (100) gives the solution $f(z)=c z^{\frac{a_{2}}{a_{2}-1}}$, where $\mathrm{c}$ is an arbitrary constant. Hence, (101) reduces to $(a-2) c=0$.

If $a_{2}=2$ then the solution of the system (100)-(103) is

$$
f(z)=c z^{2} \text { and } g(z)=4 a_{1} c z^{2}
$$

If $a_{2} \neq 2$, then $c=0$ and $f(z)=g(z)=0$, for any $z$. It is now clear that the potential symmetry $W_{5}$ yields to the solution

$$
u(x, t)=\lambda\left(2 x+a_{1}\right) \exp \left(\frac{\left(2 x+a_{1}\right)^{2}}{2}+4 t\right)
$$

for some real number $\lambda$ if $a_{2}=2$; and to the trivial solution $u(x, t)=0$ for all $x, t \in \mathbb{R}$ otherwise.

\section{Conclusions}

The approach we used to determine the point symmetries of the Fokker Planck equation (1) already exists in the literature. Given a trivial solution, one has constructed a family of solutions for this equation. The symmetry analysis of the equation (1) that we have performed highlights the fact that the potential symmetries constitute the powerful tool for solving partial differential equations provided that these are written in their conserved form. Exact solutions can be found from these types of symmetries as it is the case in the present work with the invariant surface conditions associated with the equation (1).

\section{Acknowledgements}

The authors would like to thank the Deutscher Akademischer Austaush Dienst (DAAD) for its financial support. The second author is supported by the NLAGA project and PACER II. Part of this work was done during his visit at IMSP (Port-Novo, Benin) funded by the DAAD. He expresses his gratitude and thanks to these institutions.

\section{References}

[1] Bluman G. W.; Kumei S., Symmetries and Differential Equations. Springer Verlag, New York, 1989.

[2] Brics M.; kaup `zs J.; Mahnke R., How to solve Fokker-Planck equation treating mixed eigenvalue spectrum, Condensed Matter Physics, Vol. 16, $n^{\circ} 1$ (2013) 1-13.

[3] Carrillo J. A.; Cordier S. and Mancini S., A decision-making Fokker-Planck model in computational neuroscience, J. Math. Biol., 63, pp. 801-830, (2011).

[4] Carrillo J. A.; Cordier S. and Mancini S., One dimensional Fokker-Planck re-duced dynamics of decision making models in Computational Neuroscience, Commun. Math. Sci., 11 (2), pp. 523-540, (2013).

[5] Chancelier J.-P.; Cohen De Lara M.; Pacard F., Equation de Fokker-Planck pour la densité d'un processus aléatoire dans un ouvert régulier. Comptes rendus de l'Académie des
Sciences. Série 1, Mathématique, 1995, vol. 321, $n^{\circ} 9$, pp. 1251-1256.

[6] Gardiner C., Stochastic Methods: A Handbook for Natural and Social Sciences. $4^{\text {th }}$ Ed. Springer, 2009.

[7] Hesam S.; Nazemi A. R. and Haghbin A., Analytical solution for the Fokker-Planck equation by differential transform method, Scientia Iranica, Vol. 19, Issue 4, (2012), pp. 1140-1145.

[8] Hottovy S., The Fokker-Planck Equation, http://www.math.wisc.edu/ $\quad$ shottovy/NumPDEreport.pdf, (2011).

[9] Ibragimov N. H., Transformation Groups Applied to Mathematical Physics. Reidel Publishing Company: Dordrecht, Netherlands, 1985.

[10] Jia Zheng, Lie Symmetry Analysis and Invariant Solutions of a Nonlinear Fokker-Planck Equation Describing Cell Population Growth, Advances in Mathematical Physics, https://doi.org/10.1155/2020/4975943, (2020).

[11] Lie Sophus, Theories der Transformations gruppen. Teubner, Leipzig, 3, (1893).

[12] Martin Ph. A., Introduction aux Processus Stochastiques en Physique. Nonequilibrium Statistical Mechanics, 2006.

[13] Olver P. J., Applications of Lie Groups to Differential Equations. $2^{\text {nd }}$ Ed., GTM, Vol. 107, Springer Verlag, New York, 1993.

[14] Olver P. J., Equivalence, Invariants and Symmetry, Cambridge University Press, 1995.

[15] Ouhadan A.; El Kinani E. H.; Rahmoune M. and Awane A., Symétries ponctuelles et potentielles de l'équation de FokkerPlanck. African Journal of Mathematical Physics, Vol 5 (2007) 33-41.

[16] Ouhadan A.; El Kinani E. H.; Rahmoune M.; Awane A.; Ammar A. and Essabab S., Generalized Symmetries and Some new Solution of the Fokker-Planck Equation. African Journal Of Mathematical Physics, Volume 7, No 1 (2009) 9-17.

[17] Ovsiannikov L. V., Group Analysis of Differential Equations. Academic Press, New York, 1982.

[18] Pucci E. and Saccomandi G., Potential symmetries and solutions by reduction of partial differential equations. $J$. Phys.: Math. Gen. 26 (1993) 681-690.

[19] Tanski I. A., Fundamental solution of Fokker-Planck equation., http://arxiv.org/ pdf/nlin/0407007.pdf, (2004). 
[20] Till D. F., Nonlinear Fokker-Planck equations: Fundamentals and Applications. Springer-Verlag, Berlin Heidlberg, 2005.

[21] Winter S., Group classification and symmetry reductions of a nonlinear Fokker-Planck equation based on the SharmaTaneja Mittal entropy, arXiv: 1904.01307v1 [math. AP] 2 Apr 2019.
[22] Zorzano M. P.; Mais H.; Vazquez L., Numerical Solution for Fokker-Planck Equa-tion in Accelerators. Physica D 113, (1998) 379-381. 